\title{
Study of the valence electronic density distribution in $Z=112-120$ atoms
}

\section{Mikhail Y. Kaygorodov*}

${ }^{1}$ Department of Physics, St. Petersburg State University, Universitetskaya nab., 7/9, 199034 St. Petersburg, Russia

E-mail: st031727@student.spbu.ru

\section{Yury S. Kozhedub}

${ }^{1}$ Department of Physics, St. Petersburg State University, Universitetskaya nab., 7/9, 199034 St. Petersburg, Russia

E-mail: y.kozhedubespbu.ru

\section{Ilya I. Tupitsyn}

${ }^{1}$ Department of Physics, St. Petersburg State University, Universitetskaya nab., 7/9, 199034 St. Petersburg, Russia

${ }^{2}$ Center for Advanced Studies, Peter the Great St. Petersburg State Polytechnic University, Polytekhnicheskaya 29, St. Petersburg 195251, Russia

${ }^{3}$ St. Petersburg Nuclear Physics Institute, mkr. Orlova roshcha 1, Leningrad district, Gatchina, 188300, Russia

E-mail: i.tupitsynespbu.ru

\section{Vladimir M. Shabaev}

${ }^{1}$ Department of Physics, St. Petersburg State University, Universitetskaya nab., 7/9, 199034 St. Petersburg, Russia

E-mail: v.shabaevespbu.ru

\begin{abstract}
The localization properties of the valence electronic density are considered for superheavy atoms in the range of atomic numbers $112 \leq Z \leq 120$, paying special attention to oganesson atom $(Z=$ 118). As in [P. Jerabek et al., Phys. Rev. Lett. 120, (2018) 053001], the atomic shell structure is studied by means of the relativistic calculations using a non-relativistic expression for electron localization function. The influence of the relativistic effects on the distribution of the valence electronic density is evaluated by performing related calculations in the non-relativistic limit. One-electron Dirac-Fock and Hartree-Fock valence subshell densities are calculated and no signs of smearing out these densities are found.
\end{abstract}

International Conference on Precision Physics and Fundamental Physical Constants - FFK2019 9-14 June, 2019

Tihany, Hungary

${ }^{*}$ Speaker. 


\section{Introduction}

Superheavy elements (SHEs) are usually referred to artificially synthesized elements starting from rutherfordium $(Z=104)$ up to the last known element oganesson $(Z=118)$ which closes the 8th row of the Periodic Table. It is a major challenge to study physical and chemical properties of SHEs since the elements have short half-lives and low production rates. The lack of stable isotopes and demand on specific facilities are also a great issue experiment has to cope with. Therefore, the most available way to study physical and chemical properties of SHEs nowadays remains theoretical calculations based on the most elaborated methods of relativistic quantum mechanics [1,2].

Various theoretical calculations were performed to understand and predict physical properties of SHEs such as ionization potentials, energy levels, polarizabilities, electronic affinities [3, 4, 5, $6,7]$. The calculations predict a growing impact of relativistic effects on the atomic shell structure what can lead to exotic and anomalous properties of SHEs compared with their lighter homologous. For example, in Ref. [3] it was shown that Og has a positive electron affinity, in spite of having the electronic configuration of a noble gas. This means that Og may form a negatively charged ion. Another example of anomalous properties of SHEs is a recent study of Og electronic structure in Ref. [8]. In this work it was concluded that due to relativistic effects the electron density becomes more uniformly distributed over the entire atomic range and approaches the uniform-electron gas regime in the valence region. The conclusion was based on analysis of an electron localization function (ELF), which was introduced first in Ref. [9] for the sake of visualization of the shell structure in atoms and chemical bonding in molecules.

The present work is focused on examination of the results of Ref. [8] for Og atom and more detailed study of the SHE electronic density in the valence region. The elements which have been chosen to study are Og and its "neighbors" in the Periodic Table with the atomic charge numbers $112 \leq Z \leq 120$ and the electronic configurations $[\mathrm{Rn}] 5 f^{14} 6 d^{10} 7 s^{n} 7 p^{m} 8 s^{k}$, where $0 \leq n \leq 2,0 \leq$ $m \leq 6,0 \leq k \leq 2$. We examine the shell structure yielded by the ELF for both relativistic and nonrelativistic one-electron densities obtained as the solutions of the single-configuration Dirac-Fock equations. The non-relativistic data are obtained by the non-relativistic limit $(c \rightarrow \infty, c$ is the speed of light) of the Dirac-Fock calculations. The Dirac-Fock equations were solved using the DiracFock-Sturm approach described in Ref. [10]. A non-relativistic method of the ELF evaluation, which incorporates some localized attraction potential, is also suggested. The approach allows us to obtain the ELF profile close to the relativistic one in the valence region. The relativistic and nonrelativistic electron subshell density distribution widths, which can be considered as an indicator of smearing out of the one-electron density, are studied as well.

The paper is organized as follows: in Sec. 2 the derivation of the non-relativistic expression for the ELF is given, then the work is proceeded with numerical results which are presented in Sec. 3, and, finally Sec. 4 contains some conclusions and outlook.

\section{Electron localization function}

To study one-electron localization properties in the valence region one cannot rely on the total one-electron density since strong overlapping of the shell densities makes impossible to reveal the shell structure. To extract the desired quantitative information about the shell structure one 
could exploit the Laplacian, $-\nabla^{2} \rho$, quantity $-|\nabla \rho| / \rho$ and several other functions of the total oneelectron density. The ELF is one of the instruments to perform qualitative analysis of atomic shell structure in the 3D configuration space $[9,11,12,13]$.

In the ELF the electron localization is related to the smallness of the conditional pair probability of finding a spin-like electron in vicinity of another one which in the Hartree-Fock approximation is given by $[9,11]$ :

$$
D(\boldsymbol{r})=\frac{1}{2}\left[\tau(\boldsymbol{r})-\frac{1}{4} \frac{|\nabla \rho(\boldsymbol{r})|^{2}}{\rho(\boldsymbol{r})}\right],
$$

where the one-electron density $\rho(\boldsymbol{r})$ and the kinetic energy density $\tau(\boldsymbol{r})$ are defined by

$$
\rho(\boldsymbol{r})=\sum_{j}\left|\varphi_{j}(\boldsymbol{r})\right|^{2}, \quad \tau(\boldsymbol{r})=\sum_{j}\left|\nabla \varphi_{j}(\boldsymbol{r})\right|^{2} .
$$

Here the index $j$ numerates the relativistic one-electron atomic wavefunctions $\varphi_{j}(\boldsymbol{r})$.

The dimensionless localization index $\chi$ may be represented as a ratio $\chi=\frac{D(\boldsymbol{r})}{D_{0}(\boldsymbol{r})}$, where $D_{0}(\boldsymbol{r})$ corresponds to the case, when the uniform electron gas relation between the particle density and the kinetic-energy density is considered for bound electrons in the atomic potential under consideration (Thomas-Fermi atomic model),

$$
D_{0}(\boldsymbol{r})=\frac{3}{10}(3 \pi)^{2 / 3} \rho(\boldsymbol{r})^{5 / 3}
$$

With these conventions we define the $\operatorname{ELF}(\eta(\boldsymbol{r}))$ as

$$
\eta(\boldsymbol{r})=\frac{1}{1+\chi^{2}(\boldsymbol{r})}
$$

We emphasize that for atomic configurations with closed shells $\rho(\boldsymbol{r}), \tau(\boldsymbol{r})$ and, therefore, $D(\boldsymbol{r})$ and $D_{0}(\boldsymbol{r})$ depend only on $r=|\boldsymbol{r}|$. For configurations with open shells it is not true. However, one can average $\rho(\boldsymbol{r})$ and $\tau(\boldsymbol{r})$ over angles and then consider $D(\boldsymbol{r})$ and $D_{0}(\boldsymbol{r})$ again as functions of $r$.

The ELF is normalized in such a way that the low electronic density stands for values of the ELF close to 0 and the regions of strong electron localization correspond to values of the ELF close to 1 . It should be noted that for the uniform electron gas $\rho$ is constant, the ELF $\eta=0.5$.

\section{Results}

To confirm that our definition of the ELF agrees with one used in Ref. [8], in Fig. 1a and Fig. 1b we present a comparison of the ELF plot for Og atom obtained in the present work with the ELF plot which was taken from Ref. [8]. Both figures display the ELF for Og atom for the relativistic and non-relativistic calculations. One can see perfect agreement between these two results. For the relativistic calculations the ELF in the valence region is indeed close to 0.5, but in the non-relativistic case a pronounced shell structure of Og can be observed. However, we warn the reader that in [8] the x-axis entry should be $\ln \left(r / a_{0}\right)$ instead of $\log _{10}\left(r / a_{0}\right)$.

Let us now examine the Dirac-Fock and Hartree-Fock electron subshell density distribution widths. We define the widths as the standard deviation (STD) of the corresponding subshell density distribution. It is expected that if there is some sort of smearing out of the atomic shell structure 


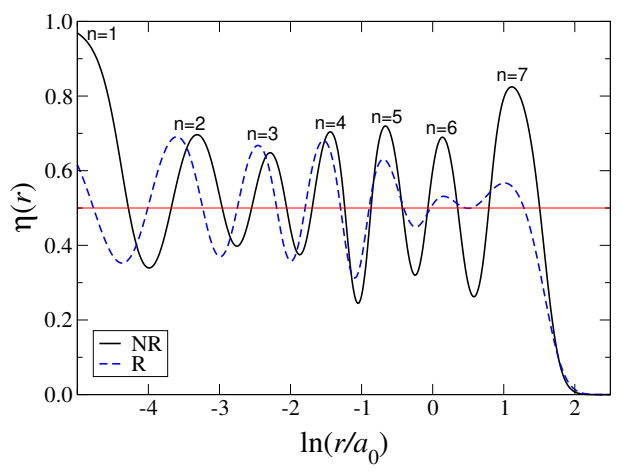

(a) Present work.

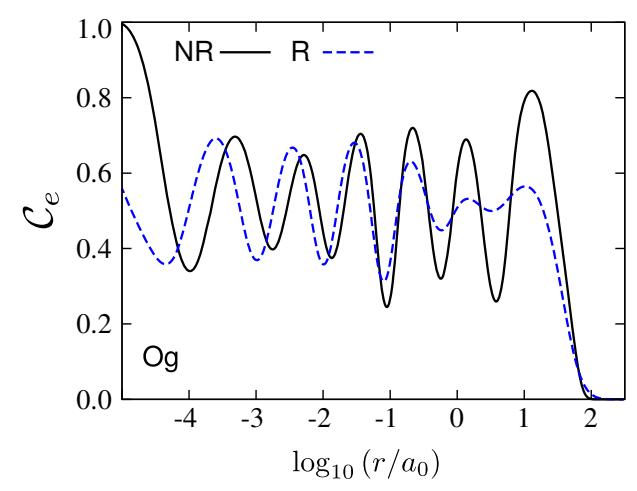

(b) Plot from Jerabek et al. [8].

Figure 1: ELFs for Og atom from the non-relativistic (NR) and relativistic (R) calculations as functions of the distance from the nucleus.

then the one-electron subshell density should be smeared out as well. For elements with the atomic charge numbers $112 \leq Z \leq 120$, in Fig. 2 we plot STD of the valence subshell electronic density distributions. However, as one can see from Fig. 2 the widths of the Dirac-Fock and non-relativistic Hartree-Fock subshells tend to be sharper with increasing atomic number. Therefore, the relativistic and non-relativistic one-particle subshell widths do not show any behavior similar to the uniform electron gas. The break in the smooth decrease of the STDs after $Z=118$ is attributed to appearance of electrons on the $8 s$ orbital.

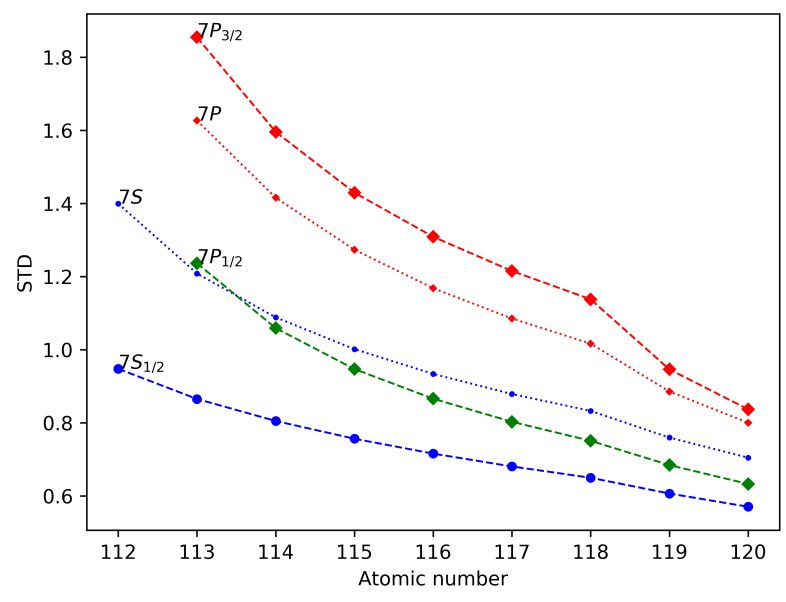

Figure 2: The standard deviations (STDs) of the valence electron subshell densities of the SHE series. The dashed lines stand for the Dirac-Fock subshell widths and the dotted lines indicate the Hartree-Fock ones.

The ELF for the sequence of atoms with the atomic charge numbers $112 \leq Z \leq 120$ is studied to comprehend whether the function predicts smearing out of the valence shell structure only for Og atom or for the other atoms as well. Fig. 3 shows the ELF in the valence region for atoms with $112 \leq Z \leq 120$ in the non-relativistic calculations. The green dots in the $Z-\ln \left(r / a_{0}\right)$ plane indicate the root-mean square (RMS) radii of the Hartree-Fock subshell electron density distribution. We 
note that in the valence region the maxima of the ELF do not coincide with the positions of the RMS radii. The distinct ELF peaks correspond to the $n=6$ and $n=7$ shells in the non-relativistic calculations. The known artifact of the ELF for atoms with only valence $n s$ orbitals occupied [9] is eliminated in the figure.

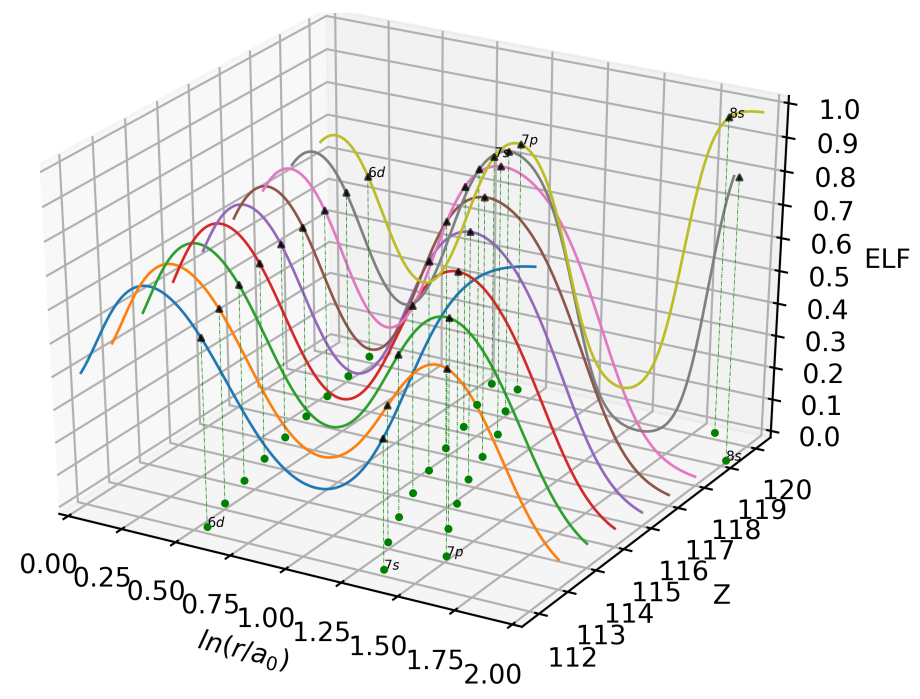

Figure 3: The ELFs for the valence region in the non-relativistic calculations for $112 \leq Z \leq 120$ elements.

The results of the relativistic calculations are presented in Fig. 4. These calculations of the ELF along the considered SHE sequence show that $\mathrm{Og}$ atom has the most pronounced shell structure in the valence region. The incongruity of the RMS radii of the Dirac-Fock subshells and the ELF peaks is observed in the relativistic calculations as well. The relativistic calculations give values of the ELF close to 1 for atoms with $Z=119$ and $Z=120$, where the $8 s$ orbital is occupied. However, the ELF for the $Z=112$ element, where the $7 s$ orbital is fully occupied, does not show this behavior.

To investigate whether the behavior of the ELF in the relativistic calculations is indeed due to strong relativistic effects we simulate the contraction of the valence $s$ and $p$ orbitals by incorporating a highly localized attraction potential into the calculations of the $7 s$ and $7 p_{1 / 2}$ states within the non-relativistic limit $(c \rightarrow \infty)$ of the Dirac-Fock equations. In these calculations all electrons become non-relativistic, and the $7 s$ and $7 p_{1 / 2}$ states are shifted closer to the nucleus. The attraction potential was taken in the form (in a.u.)

$$
V_{\text {Att.Pot. }}(r)=-22500 \mathrm{e}^{-140 r} .
$$

This form is chosen to get the simplest strongly localized potential for the states under consideration. Fig. 5 demonstrates how the described incorporation of $V_{\text {Att.Pot. }}$ into the non-relativistic calculations changes the shape of the ELF for Og atom. In the plot the relativistic (blue dashed line), non-relativistic (black solid line) and non-relativistic with $V_{\text {Att.Pot. (orange dotted line) ELFs }}$ are shown. The profile of the ELF in the non-relativistic calculations with $V_{\text {Att.Pot. }}$ in the valence region is close to the profile of the ELF in the relativistic calculations. This means that only the 


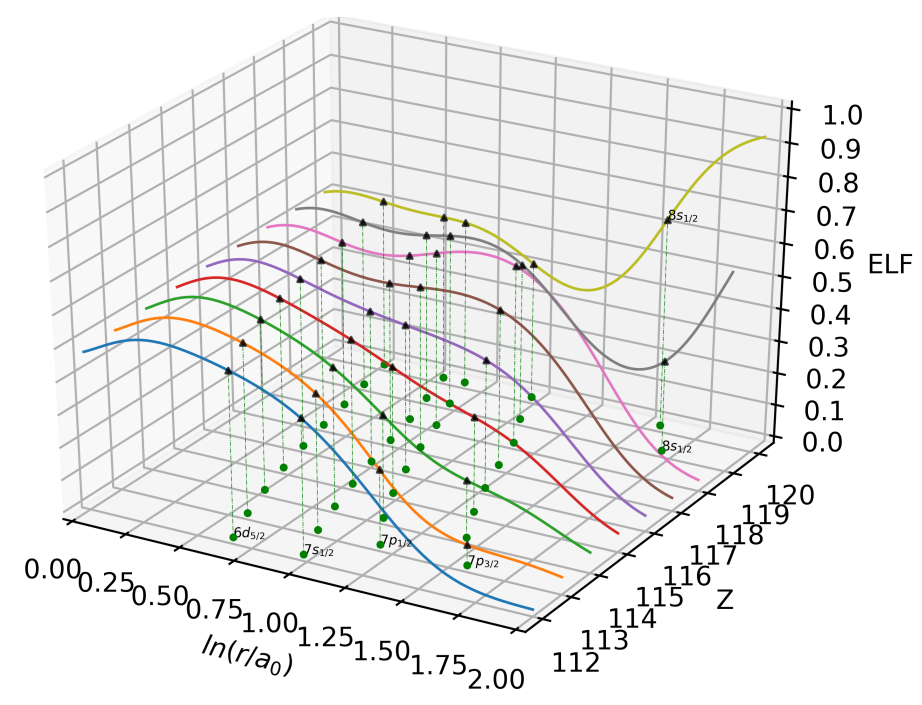

Figure 4: The ELFs for the valence region in the relativistic calculations for $112 \leq Z \leq 120$ elements.

contraction of the $7 s$ and $7 p_{1 / 2}$ orbitals, which leads to the shift of the $7 s$ and $7 p_{1 / 2}$ orbitals closer to the main maxima of the outer core $6 d$ orbital, is responsible for the fact that the ELF is close to 0.5 in the valence region.

Although all the results presented have been obtained within the framework of the singleconfiguration Dirac-Fock method, we also have studied the role of the configuration-interaction (CI) effects on the smearing out of the valence shell densities in Og atom. It is known that these effects can be very important in calculations of various properties of SHEs [14]. The CI-DF calculations of the electronic structure of $\mathrm{Og}$ show, however, that the $\mathrm{CI}$ weight of the ground configuration $[\mathrm{Rn}] 5 f^{14} 6 d^{10} 7 s^{2} 7 p^{6}$ is about $94 \%$. This means that other configurations do not give any noticeable contributions despite the supposed "smearing out" of the valence shells.

\section{Conclusion and Outlook}

The present work was aimed at studying the electron localization properties of the valence electron density for SHEs with the atomic charge numbers $112 \leq Z \leq 120$. We investigated in details the results based on the ELF expression [9] for these elements. It was found that the relativistic calculations predict smearing out of the valence electronic density for all SHEs under consideration, while the non-relativistic calculations yield a distinct structure of atomic orbitals. In contrast to the ELF results, the calculations of the one-electron Dirac-Fock and non-relativistic HartreeFock electron subshell density widths do not indicate any signs of smearing out of the electron density distribution. The widths remain finite and with increasing the atomic number they become even smaller. If there were a case of delocalization in the valence region, then the valence oneelectron density would be smeared out as well. To study the origin of smearing out of the ELF in the relativistic calculations, we have also performed the following test. In the non-relativistic calculations, where the ELF predicts a sharp shell structure, we artificially inserted a local attraction potential into the equations for the valence $s$ and $p$ orbitals. This procedure simulates the relativis- 


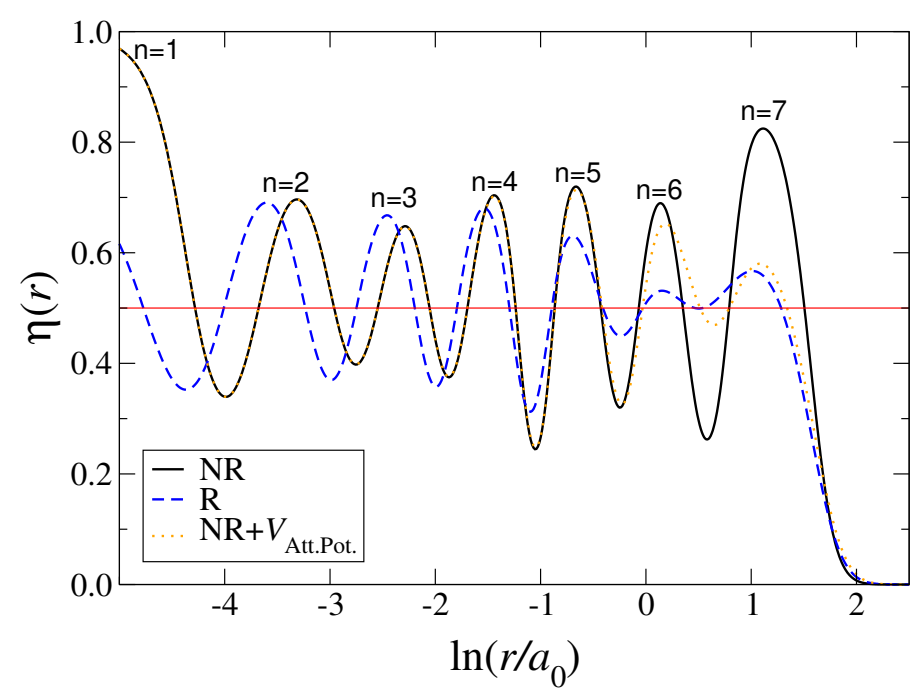

Figure 5: The ELFs in the relativistic (R), non-relativistic (NR) and non-relativistic with attraction potential $\left(\mathrm{NR}+V_{\text {Att.Pot. }}\right)$ calculations for $\mathrm{Og}$ atom.

tic contraction of the considered orbitals. The resulted ELF in the valence region has almost the same profile as the ELF in the relativistic calculations. Based on these results, we conclude that the smearing out of the ELF is due to the shifts of the $7 s$ and $7 p_{1 / 2}$ orbitals towards the $6 d$ orbital caused by the relativistic effects.

\section{Acknowledgments}

This work is supported by the Russian Foundation for Basic Research (RFBR) (Grants No. 1803-01220, 17-02-00216) and by SPbSU-DFG (Grants No. 11.65.41.2017, No. STO 346/5-1). M.Y.K. and V.M.S. acknowledge the support of the foundation for the advancement of the theoretical physics and mathematics "Basis". I.I.T. thanks the Ministry of Education and Science of the Russian Federation (Grant No. 3.1463.2017/4.6) and St. Petersburg State University (Grant No. 37717909).

\section{References}

[1] V. Pershina, Electronic structure and properties of superheavy elements, Nucl. Phys. A 944 (2015) 578.

[2] E. Eliav, S. Fritzsche and U. Kaldor, Electronic structure theory of the superheavy elements, Nucl. Phys. A 944 (2015) 518.

[3] E. Eliav, U. Kaldor, Y. Ishikawa and P. Pyykkö, Element 118: The First Rare Gas with an Electron Affinity, Phys. Rev. Lett. 77 (1996) 5350.

[4] V. Pershina, A. Borschevsky, E. Eliav and U. Kaldor, Adsorption of inert gases including element 118 on noble metal and inert surfaces from ab initio Dirac-Coulomb atomic calculations, J. Chem. Phys. 129 (2008) 144106. 
[5] V. A. Dzuba, Ionization potentials and polarizabilities of superheavy elements from $\mathrm{Db}$ to $\mathrm{Cn}$ (Z=105-112), Phys. Rev. A 93 (2016) 032519.

[6] J. S. M. Ginges and V. A. Dzuba, Spectra of barium, radium, and element 120: Application of the combined correlation-potential, singles-doubles, and configuration-interaction ab initio methods, Phys. Rev. A 91 (2015) 042505.

[7] B. G. C. Lackenby, V. A. Dzuba and V. V. Flambaum, Atomic structure calculations of superheavy noble element oganesson (Z=118), Phys. Rev. A 98 (2018) 042512.

[8] P. Jerabek, B. Schuetrumpf, P. Schwerdtfeger and W. Nazarewicz, Electron and Nucleon Localization Functions of Oganesson: Approaching the Thomas-Fermi Limit, Phys. Rev. Lett. 120 (2018) 053001.

[9] A. D. Becke and K. E. Edgecombe, A simple measure of electron localization in atomic and molecular systems, J. Chem. Phys. 92 (1990) 5397.

[10] I. I. Tupitsyn, V. M. Shabaev, J. R. Crespo Löpez-Urrutia, I. Draganič, R. Soria Orts and J. Ullrich, Relativistic calculations of isotope shifts in highly charged ions, Phys. Rev. A 68 (2003) 022511.

[11] A. Savin, O. Jepsen, J. Flad, O. K. Andersen, H. Preuss and H. G. von Schnering, ELF: The Electron Localization Function, Angew. Chem. Internat. Ed. Eng. 31 (1992) 187.

[12] M. Kohout and A. Savin, Atomic shell structure and electron numbers, Int. J. Quantum Chem. 60 (1996) 875 .

[13] A. Savin, R. Nesper, S. Wengert and T. F. Fässler, ELF: The Electron Localization Function, Angew. Chem. Internat. Ed. Eng. 36 (1997) 1808.

[14] B. G. C. Lackenby, V. A. Dzuba and V. V. Flambaum, Theoretical calculation of atomic properties of superheavy elements $Z=110-112$ and their ions, arXiv:1910.01414 [physics] (2019) . 